\title{
Studies on Reflector Materials for Cold Neutrons
}

\author{
J. Rolando Granada ${ }^{1, *}$, J. Ignacio Márquez Damián ${ }^{2}$, and Christian Helman ${ }^{1}$ \\ ${ }^{1}$ Centro Atómico Bariloche and Instituto Balseiro, 8400 S.C. de Bariloche (RN), CNEA, Argentina \\ ${ }^{2}$ Centro Atómico Bariloche and Instituto Balseiro, 8400 S.C. de Bariloche (RN), CONICET, Argentina
}

\begin{abstract}
Neutron scattering techniques and neutron applications in general are powerful and wellestablished tools for research in science and technology. However, those are highly intensity-limited techniques and the need to reduce the neutron loses between their source to detection is then an imperative endeavor. In particular, great effort has been devoted to the development of efficient ways to transport and guide neutron beams, as well as to reduce the flux leakage in the non-emitting sides of moderator systems. In this work we investigate and confirm by calculation in a real material the great quality of diamond nanoparticles as reflector for ultra-cold and very-cold neutrons, and propose the use of Magnesium Hydride at a low temperature as a very good reflector for cold neutrons
\end{abstract}

\section{Introduction}

The tremendous potential of neutrons as a probe of matter or as a research object by itself is limited by the relatively low-flux intensity of the neutron sources, as compared with photon sources. In addition, the different processes (production, slowing-down) and devices (moderators, transport systems, collimators, energyselectors, detectors, etc.) reduce by several orders of magnitude the actual neutron intensity that eventually conveys the experimental information of interest. One of the research lines devoted to minimize those loses has been oriented to the search for efficient reflector materials, that may improve the efficiency of guiding surfaces or the actual reflection of neutrons on a containment walls to reduce their leakage. A large body of work has been done in the past, particularly concerning the interaction of slow neutrons with diamond nanoparticles. It has been demonstrated the high reflectivity of this material for $\mathrm{UCN}$ and $\mathrm{VCN}$ on the basis of calculations for ideal systems as well as by scattering experiments, and proposed that such capacity may extend to higher neutron energies, thus bridging the "reflectivity gap" in the neutron spectrum $[1,2]$.

In this work we present calculations aimed at evaluating the thermal neutron cross sections of bulk diamond and the small angle scattering contribution due to the nanostructure, using recent experimental information. With those ingredients we generated a cross section library over an extended neutron energy range for a real nanodiamond (ND) powder. Reflectivity calculations using our library confirm the excellent performance of that material for ultra-cold and verycold neutrons, and also its rapid decrease for neutron velocities larger than about $150 \mathrm{~m} / \mathrm{s}$.
A different approach was employed to explore the characteristics of potential cold neutron reflectors, based on the requirement of a large scattering cross section combined with a small value of the average cosine of the scattering angle over the energy range of interest. Those conditions led us to consider metal hydrides, and eventually select magnesium hydride as a good candidate to fulfill those requirements.

\section{Diamond nanopowders}

During the last two decades, Nesvizhevsky and coworkers have been developing a large body of calculated and experimental results concerning the use of diamond nanopowder as a very good reflector for ultra- and very-cold neutrons $[3,4]$ and its capacity as a good quasi-specular reflector for cold neutrons [5,6]. The calculations of cross sections were also done [7] for ideal ND systems always in the first Born approximation, and experimental SANS results were obtained for typical detonation nanodiamonds [8]. More recently, a research project [9] involving several groups was established aiming at a complete characterization of those materials and determination of their neutronic properties. One of those groups was able to perform SANS and transmission measurements, thus producing the first experimental total cross section results for a diamond nanopowder over the cold neutron energy region [10].

\footnotetext{
* Corresponding author: granada@cnea.gov.ar
} 


\subsection{Bulk diamond}

The description of the neutron cross sections of nanodiamond (ND) over the thermal and cold energy regions demands a proper account of the bulk diamond properties, as their contribution will be significant for thermal neutrons.

In order to evaluate the elastic and inelastic components of the cross sections, we first calculated the phonon density of states (PDOS) of diamond, using ab initio algorithms $[11,12]$. The result is shown in Fig.1, which is in good agreement with previous data $[13,14]$; also, calculations of the specific heat based on our PDOS are in excellent agreement with the experimental results of A.C. Victor and W. DeSorbo $[15,16]$.

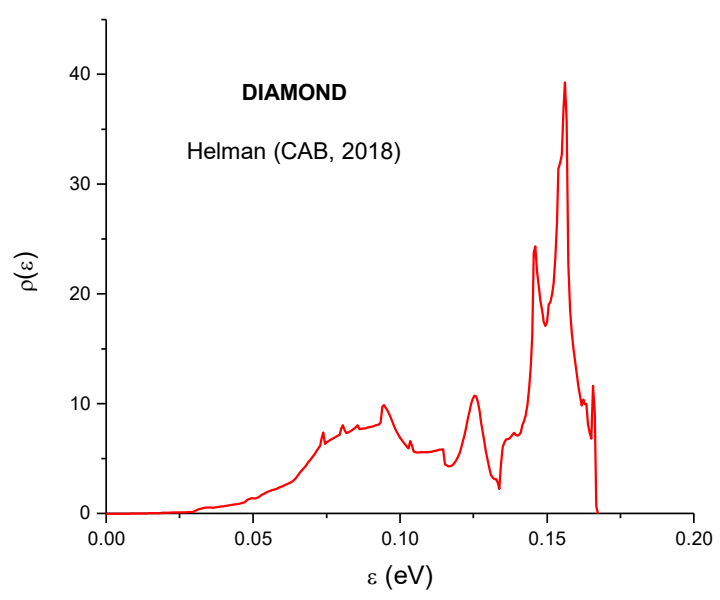

Fig. 1: Our calculated phonon density of states of diamond obtained by DFT calculation.

We developed a scattering kernel and generated cross section libraries over the thermal and cold neutron energy ranges, using the codes NJOY [17] for the inelastic contributions and NCrystal [18] for the elastic ones. The bound-atom scattering and absorption cross section values were taken from Ref.[19]. The calculated total cross section of polycrystalline bulk diamond at room temperature (heavy line) and their components (thin dashed line: elastic; thin continuous line: inelastic) are displayed in Fig.2, but surprisingly we found no experimental results for this magnitude to compare with.

\subsection{Nano diamond}

The characteristics of the diamond nanopowder and the interactions of slow neutrons with such system are given in [7]. The diffraction of the neutron wave on structural and density inhomogeneities gives rise to coherent elastic effects that superimpose upon the bulk crystal cross sections.

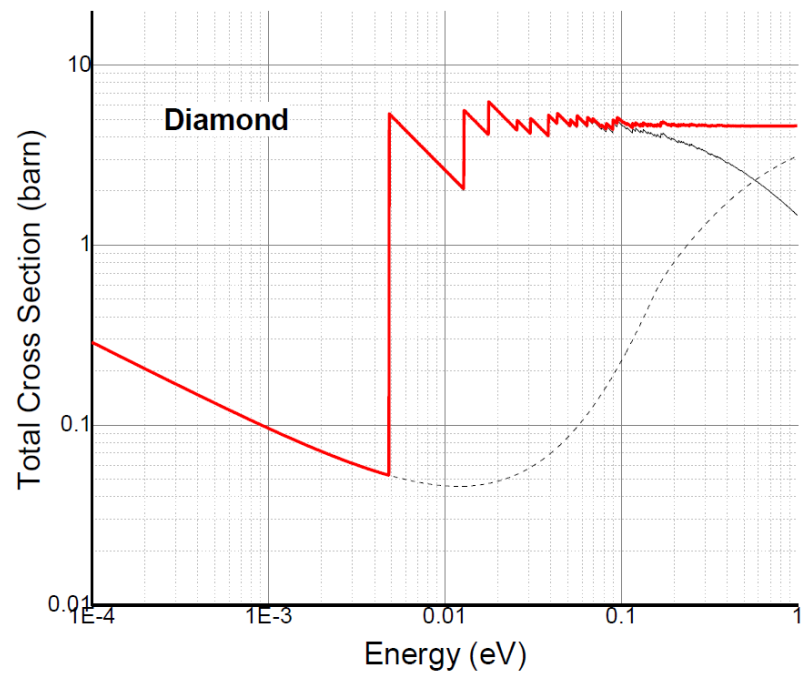

Fig. 2: The total cross section of bulk diamond over the thermal neutron energy range (red line). The black dashed line denotes the inelastic contribution, whereas the black solid line represents the elastic coherent component.

In order to produce a library corresponding to a real material, we used the experimental SANS results obtained by Teshigawara and coworkers [10], and fit them using the unified exponential/power-law approximation [20] as expressed in Eq.3 of Ref. [8]. In Fig.3 we show the results obtained for the scattered intensity $\mathrm{I}(\mathrm{Q})$ comparing the data points [10] with the fitted curve. This structure factor can be integrated to give the scattering total cross section corresponding to the coherent elastic effects caused by the nanopowder which, when added to the nanospheres' bulk diamond contributions produces the total scattering cross section for this system with apparent radius of gyration of 2.5 nm. Those calculated contributions together with the experimental points [10] are shown in Fig.4.

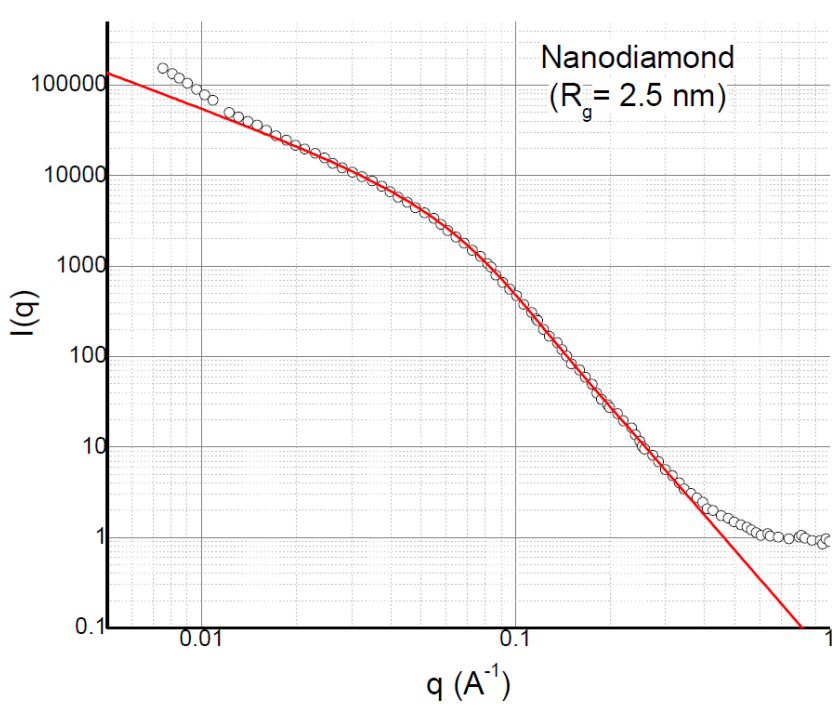

Fig. 3: Small angle scattering intensity as experimentally determined by Teshigawara et al. [10] (open circles) and our fitted curve using the unified exponential/power-law approximation [20]. 


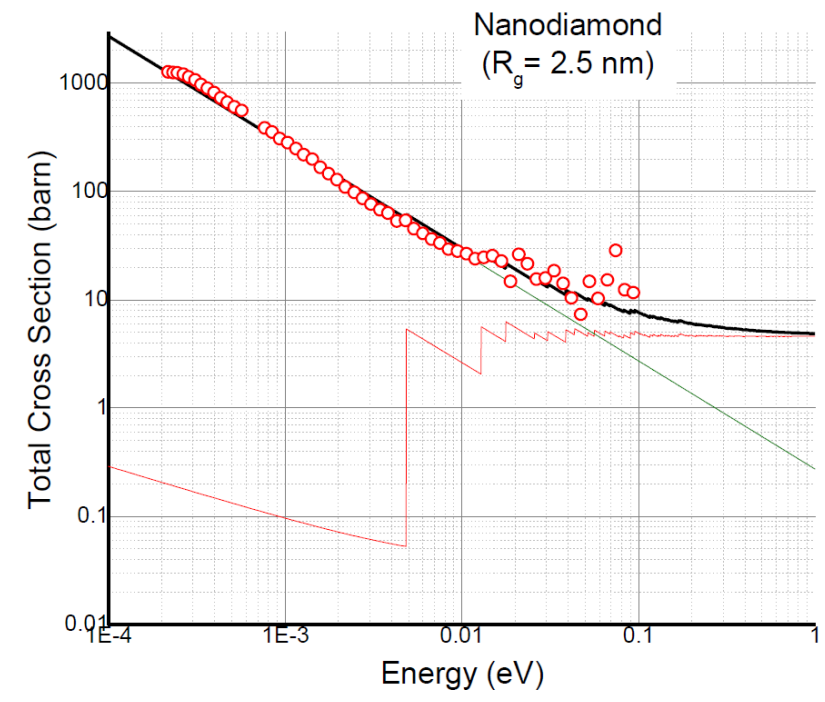

Fig. 4: Total cross section of nanodiamond, comparing our calculated curve (black line) with experimental data from Ref.[10]. Red and green lines denote the crystal and SANS components, respectively.

\subsection{ND reflectivity}

The scattering kernel, including small angle scattering effects, was implemented in the Monte Carlo program OpenMC [21], and this tool was used to study the reflective properties of nanodiamond layers. OpenMC was modified to include the total coherent scattering cross section component caused by SANS, and to sample the scattering angle from an I(Q) distribution parameterized as a two part power law.

Using this tool we modeled the experiments performed by Nesvizhevsky et al. [4], with a monoenergetic beam of neutrons impinging on a plate of variable thickness (Fig. 5). The reflectivity was computed as the ratio between the backscattered neutron current and the incident neutron current.

The results (Fig. 6) show a good agreement with experimental results, showing a good reflectivity for very cold neutrons that falls sharply for energies above $0.1 \mathrm{meV}$. Calculation of thicker ND slabs and removal of impurities allowed a modest increase of the energy at which the reflectivity decreases, but none of the calculations showed high reflectivities above $0.5 \mathrm{meV}$, in contrast of the calculations by Nesvizhevsky [2, 4].

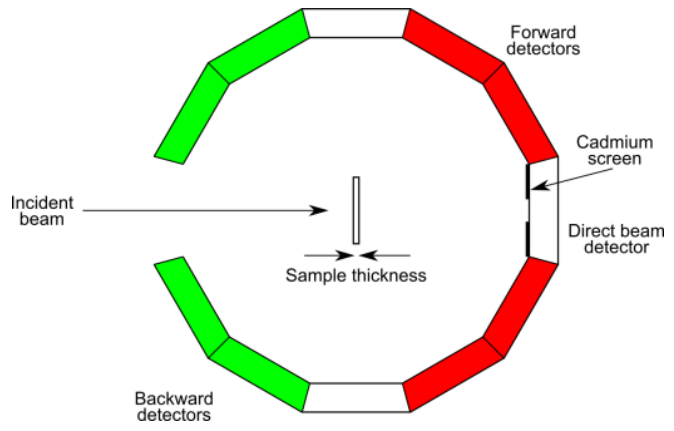

Fig. 5: Scheme of the modeled experiment. The sample thickness is the main parameter to analyse.

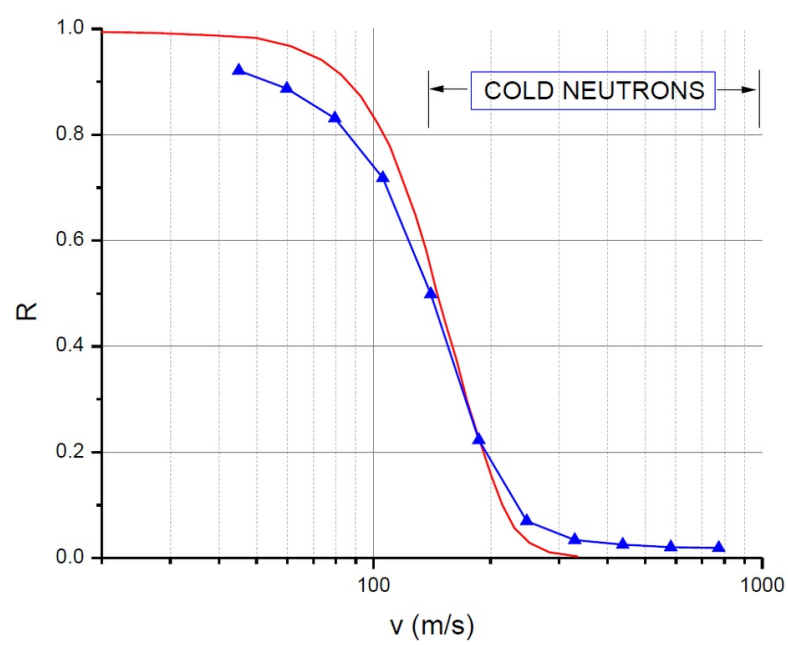

Fig. 6: Reflectivity vs. neutron velocity, for a $10 \mathrm{~mm}$ thick ND sample. The continuous line (red) is the result from Ref. [4], while the curve with symbols (blue) corresponds to our calculation $\left(\right.$ density $\left.=0.6 \mathrm{~g} / \mathrm{cm}^{3}\right)$.

\section{Magnesium hydride}

As shown in the previous section, the reflectivity of ND reduces rapidly towards the cold and thermal neutron regions, as compared to that one presented for ultra- and very-cold neutrons. This is due to the fact that when the characteristic size of the scattering object is much larger than the radiation wavelength, in this case the neutron wavelength, the coherent scattering from individual objects occurs at small angles, and the probability for backward reflection becomes very small, although specular reflection processes can still be very intense.

At neutron energies where coherent elastic interaction with the scattering system cannot produce an intensity much larger than the bound-atom coherent cross section value (see Fig.4 for diamond), other kind of interactions should be explored as mechanisms to produce a significant reflection capacity. To drive our search for a material with such property, we must look for: a) large scattering cross section, b) large elastic cross section, c) highly isotropic angular cross section, d) small inelastic cross section, and e) small absorption cross section. These requirements can be realized by the following options: a) hydrogen, b) within a solid, c) with large effective mass, d) at low temperatures, e) with an absorption cross section due only to hydrogen. Several years ago we proposed that a material with these properties is Magnesium Hydride $\left(\mathrm{MgH}_{2}\right)$ at a low temperature [22], because $\mathrm{Mg}$ has the smallest absorption cross section amongst the common metals forming hydrides [19].

\subsection{Cross sections of $\mathrm{MgH}_{2}$}

The structure and dynamics of the material are the necessary pieces of information to generate a scattering kernel and a cross section library. In Fig. 7 we show our 
adopted frequency spectrum for $\mathrm{MgH} \quad 2$ (line), together with experimental results obtained by Santisteban et al. [23] and Kolesnikov et al. [24]. We then calculated the components due to the metallic lattice and the hydrogen, employing again NJOY [17] and CRIPO [25].

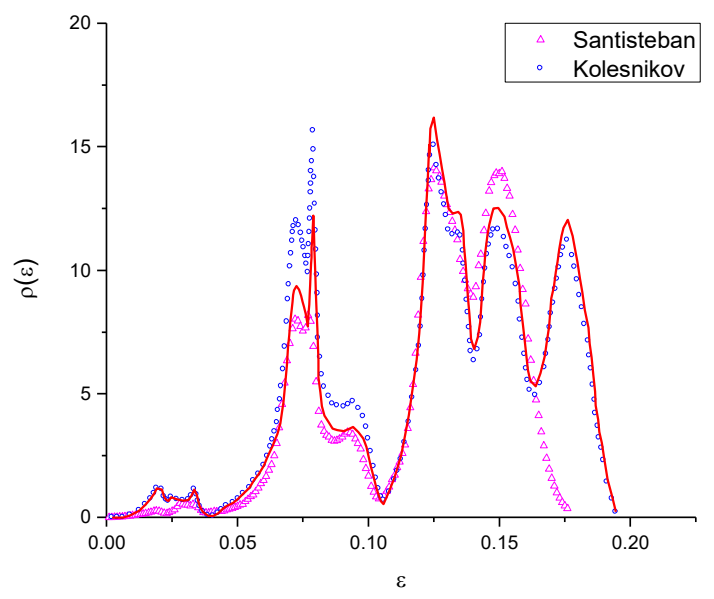

Fig. 7: Adopted density of states for $\mathrm{MgH}_{2}$ (solid line), compared with the experimental results (symbols) of Refs.[23] and [24].

As a first validation test we compared the calculated total cross section with experimental data at room temperature [26], obtaining an excellent agreement as shown in Fig. 8. The elastic and inelastic (up and down) scattering components of the total cross section of $\mathrm{H}$ in $\mathrm{MgH}_{2}$ at $20 \mathrm{~K}$ is displayed in Fig.9, to emphasize the large area where elastic processes dominate the interaction with cold neutrons. This characteristic behaviour is due to the absence of low-energy excitation modes as displayed in Fig. 7, that causes the inelastic down-scattering processes to be reduced rapidly below thermal neutron energies. In addition, at low temperatures the small-weight acoustic modes are unpopulated, and so the up-scattering processes are very much reduced.

A simple figure of merit (FoM) to gauge the potentiality of a given material as a good neutron reflector is the product of its scattering capacity by the probability of a large scattering angle in the process. Specifically, we defined

$$
\mathrm{FoM}=\sigma_{\mathrm{el}} *(1-\mu)
$$

where $\sigma \mathrm{el}$ is the total elastic cross section and $\mu$ is the average cosine of the scattering angle. This magnitude, which is essentially the transport cross section, is displayed in Fig. 10 for both $\mathrm{ND}$ and $\mathrm{MgH}_{2}$ at $20 \mathrm{~K}$. It is apparent that this FoM is larger for the hydride at neutron energies above $0.0001 \mathrm{eV}$, in a large extent originated by $\mu \sim 1$ for ND corresponding to the onset of quasi-specular reflection over that energy region.

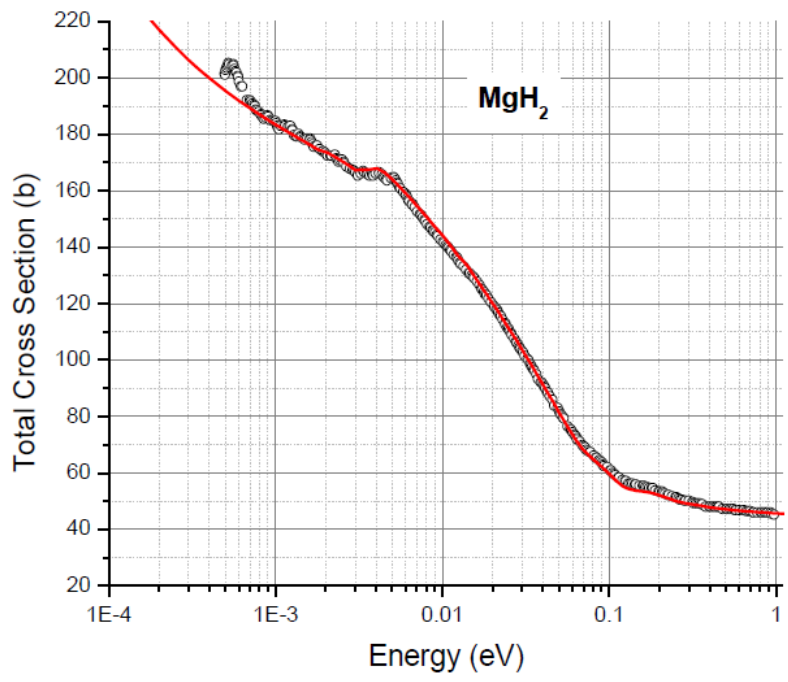

Fig. 8: Calculated total cross section of room temperature $\mathrm{MgH}_{2}$ (solid line) and the experimental points of Ref.[26].

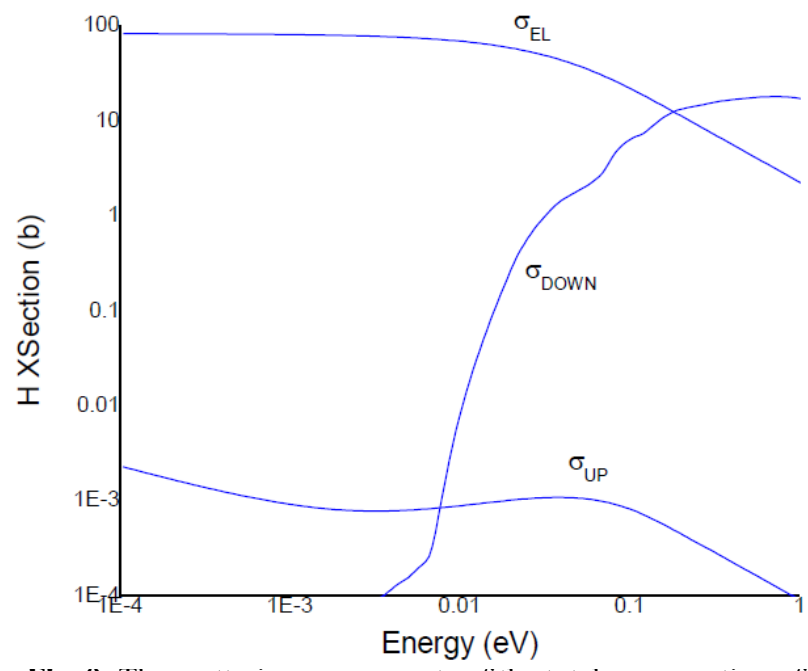

Fig. 9: The scattering components of the total cross section of $\mathrm{H}$ in $\mathrm{MgH}_{2}$ at $20 \mathrm{~K}$.

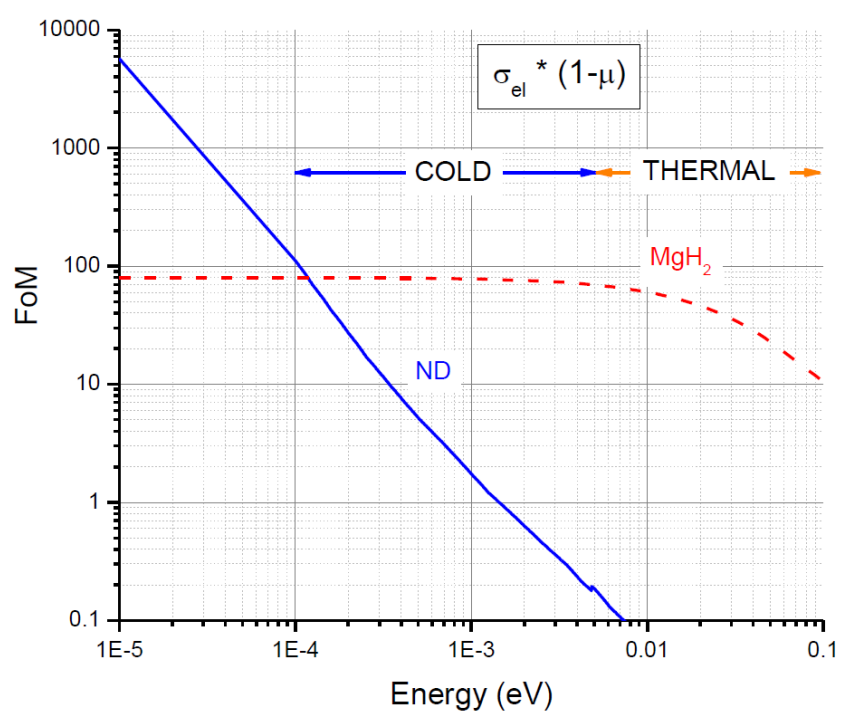

Fig. 10: Comparison of the indicated figure of merit for nanodiamond and $\mathrm{MgH}$ neutron energy range. 


\section{$3.2 \mathrm{MgH}_{2}$ reflectivity}

OpenMC was used to simulate the configuration used by Nesvizhevsky [4] (Fig. 5) to compare the reflectivity of nanodiamonds and magnesium hydride. The scattering kernel calculated with NJOY and formatted into a standard ACE library was used to model the material.

The results (Fig. 11) show a reflectivity profile which remains almost constant at all energies, with a value of $\sim 50 \%$ for $10 \mathrm{~mm}$ thickness with an apparent density of $1.0 \mathrm{~g} / \mathrm{cm}^{3}$. For very cold neutron energies the absorption of hydrogen reduces the reflectivity, but the large and almost constant elastic scattering cross section of hydrogen (Fig. 9) maintains a high reflectivity from very cold to thermal energies.

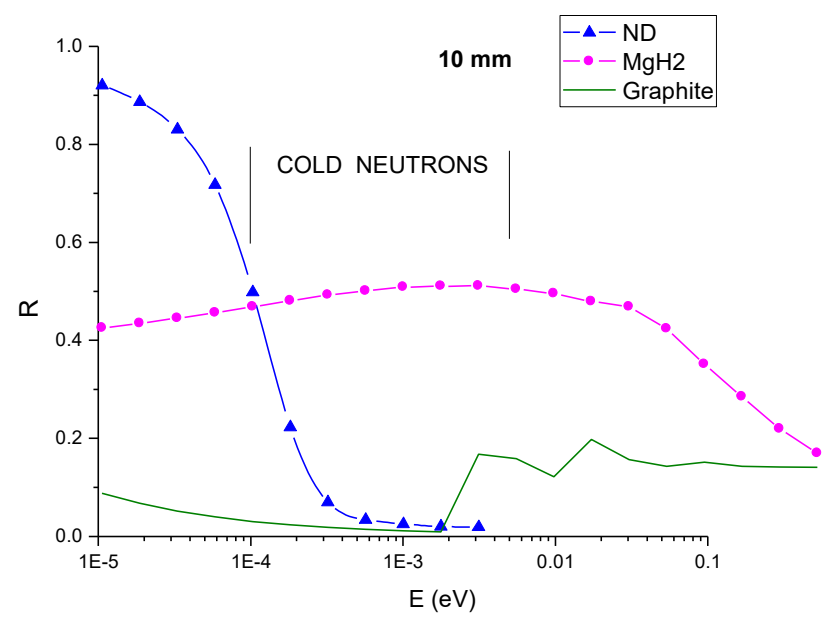

Fig.11: Reflectivity profile of $\mathrm{MgH}_{2}$. Comparative with known moderators/reflectors shows constant performance for a wide energy range.
Using this system we computed the outgoing current for a monoenergetic $1 \mathrm{MeV}, 20 \times 20 \mathrm{~cm}$ distributed source impinging on the top for four configurations:

- No premoderator, no cold neutron reflector (5 $\mathrm{mm}$ void between the cold neutron source and the beryllium block).

- $2 \mathrm{~cm}$ premoderator, no cold neutron reflector.

- $2 \mathrm{~cm}$ premoderator, $5 \mathrm{~mm}$ nanodiamond cold neutron reflector.

- $2 \mathrm{~cm}$ premoderator, $5 \mathrm{~mm} 20 \mathrm{~K}$ magnesium hydride cold neutron reflector.

As expected, the use of a premoderator produces a significant improvement in the output of the cold neutron source (Fig. 13a), but little additional gain is observed when the nanodiamond reflector is introduced except for the very cold neutron energy range. When the nanodiamond reflector is replaced by a layer of cold magnesium hydride, a significant increase in the cold neutron output is observed. Computing the ratio of the spectra to the unreflected case (Fig. 13b) this gain is quantified to $20-25 \%$ over the cold and thermal energy ranges.

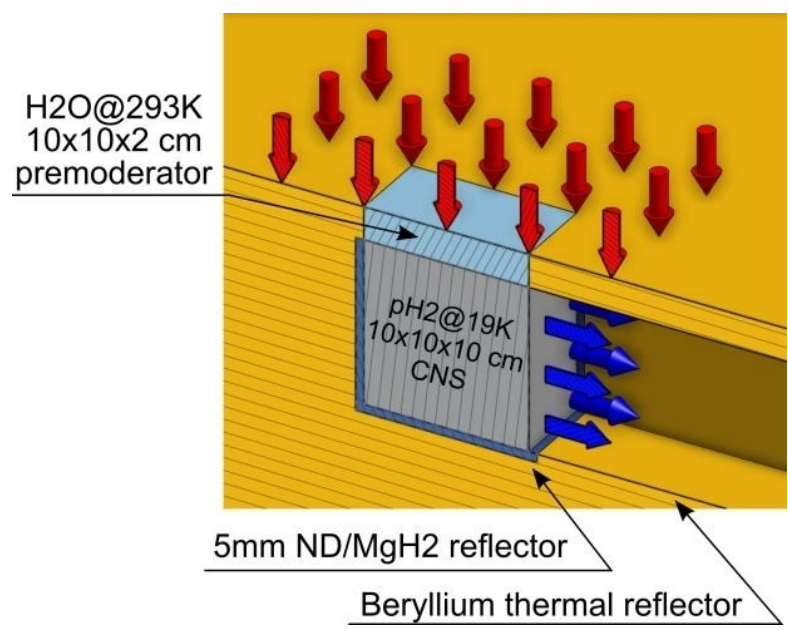

Fig.12: Geometry used to test the efficiency of nanodiamods and cold magnesium hydride as reflectors.

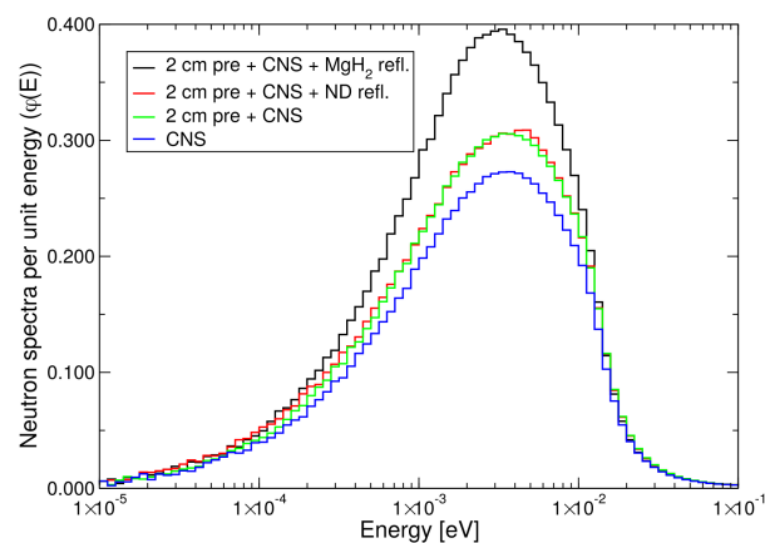

(a) 


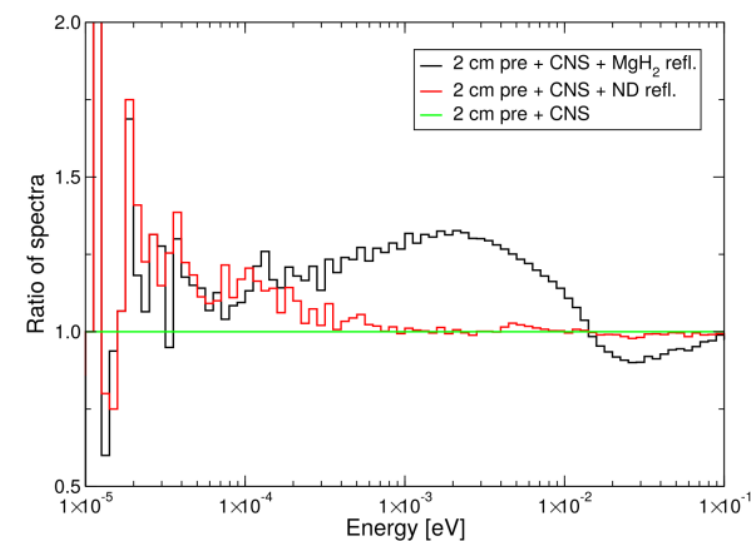

(b)

Fig.13: Spectra of the outgoing neutron current from the cold neutron source model (a). Ratio of the spectra, using as reference the case with premoderator and without cold neutron reflector (b).

\section{Conclusions}

We have developed a new scattering kernel to describe the interaction of slow neutrons with diamond, with special reference to nanopowders, and generated a cross section library that includes both the bulk contribution as well as the elastic coherent one due to the nanostructure. Reflectivity calculations using our library confirmed the excellent performance of that material for ultra-cold and very-cold neutrons.

On the other hand, to deeply explore a proposal we made few years ago concerning a potential good cold neutron reflector, we produced a new scattering kernel for $\mathrm{MgH}_{2}$ which was validated against available transmission experiments. A cross section library for this material at low temperatures was generated, and used to perform reflectivity calculations that confirm its expected good performance as reflector of cold and thermal neutrons. An experiment to determine the intensity at backscattering angles for neutrons over that energy range is being prepared at this time.
We expect that this new reflector system could help to improve the efficiency of advanced cold neutron sources, at pulsed or stationary facilities.

\section{References}

[1] E.V. Lychagin et al., Nuclear Instruments and Methods in Physics Research A 611 (2009) 302

[2] V. V. Nesvizhevsky et al., Carbon 130 (2018) 799e805

[3] V. V. Nesvizhevsky, Phys. Atomic Nuclei 65 (2002) 400

[4] V. V. Nesvizhevsky et al., Nuclear Instruments and Methods in Physics Research A 595 (2008) 631

[5] R. Cubitt et al., Nuclear Instruments and Methods in Physics Research A 622 (2010) 182

[6] V. V. Nesvizhevsky et al., Phys.Rev. A97, 023629 (2018)

[7] V. A. Artemèv et al., Crystallography Reports 61 (2016) 84

[8] M. V. Avdeev et al., J.Phys.Cond.Matter 25 (2013) 445001

[9] IAEA CRP

[10] M. Teshigawara et al., Nuclear Instruments and Methods in Physics Research A 929 (2019) 113

[11] P. Giannozzi et al., J.Phys.:Condens.Matter 21, 395502 (2009)

[12] P. Giannozzi et al., J.Phys.:Condens.Matter 29, 465901 (2017)

[13] P. Pavone et al., Phys.Rev. B48, 3156 (1993)

[14] A. Bosak and M. Krisch, Phys.Rev. B72, 224305 (2005)

[15] A. C. Victor, J.Chem.Phys. 36, 1903 (1962)

[16] W. DeSorbo, J.Chem.Phys. 21, 876 (1953)

[17] Macfarlane, R. et al LANL Report LA-UR-17-20093 (2017)

[18] Cai, X.X. and Kittelmann, T. NCrystal: A library for thermal neutron transport. Comp. Phys. Comm. (in press).

[19] F. Fernández Alonso and D. Price (Eds.) Neutron Scattering, Vol 44, Appendix (2013)

[20] G. Beaucage, J.Appl.Crystallogr. 29, 134 (1996)

[21] Paul K. Romano, Nicholas E. Horelik, Bryan R. Herman, Adam G. Nelson, Benoit Forget, and Kord Smith, "OpenMC: A State-of-the-Art Monte Carlo Code for Research and Development," Ann. Nucl. Energy, 82, 90-97 (2015).

[22] J.R. Granada et al., ICANS XX, March 4-9, 2012 (Bariloche, Argentina)

[23] J.R. Santisteban et al. Phys.Rev. B62, 37 (2000)

[24] A.I. Kolesnikov et al., J.Alloys Comp. 509S (2011) S599

[25] F. Kropff and J.R. Granada, "CRIPO: a computer code to evaluate the cross section of Polycrystalline materials", Unpublished (1974).

[26] G. Muhrer et al., Nuclear Instruments and Methods in Physics Research A 629 (2011) 251 